\title{
CARACTERISTIQUES DES REGULATEURS
}

\author{
par M. ALMERAS, \\ Ingénieur aux Ateliers NEYRPIC, \\ Prolesseur à l'Ecole des Ingénieurs Hydrauliciens de Grenoble
}

\section{INTRODUCTION}

Depuis quelques amnées la question de la régulation des turbines hydroélectriques semble être à l'ordre du jour. De nombreux articles el ouvrages traitant de cette question ont paru récemment (i). Ce n'est pas pur hasard que cette question se trouve ainsi au premier plan des préoccupations du constructeur et des exploitants. A la suite de la construction de grosses centrales de basses chutes, on a constaté que certaines installations ne pouvaient alimenter un réseau restreint a pleine charge. Comme la construetion de nouvelles centrales de basses chutes, de grande puissance, était prévuc, on a cherché à expliquer ces phénomenes. El on a trouvé l'explication dans l'inertie du fluide moteur, l'eau, caractérisée par le paramètre $(\Theta=\Sigma \mathrm{LV} / g \mathrm{H})$. Or ce paramètre, compris entre 1 et 1,5 dans les installations courantes, passe à 2 , 3 et même 4 dans certaines installations projetées ou récentes.

En dehors d'installations de basses chutes (pour lesquelles $\Theta$ est grand par suite de la petitesse de la chute $H$ ), on préroit des usinas de tres hautes chutes pour lesquelles le parametre o d'Allievi est petit, le temps $\Theta$ relalivement grand (pour des installations de hate chute) par suite de la vitesse inhabituelle de l'eau dans les conduites forcées (juscu'à $8 \mathrm{~m}$. seconde).

Dans le mème temps que le paramètre $\Theta$, caractéristique de l'inerlie du fluide moteur, algmentait, il se trouvait que le temps caractirisant l'inertie des masses lournantes (temps

(1) Bibliographie : Gabex : « Considerations sur lo probleme de stabilite », Ed. La Concorde - Lansanne. *Houille Blanche, 1946, 18 " 5 .

Srers: «Schweizerische Bauzeitung », Septembre $194 \%$.

Alǘnas : « Tonille Blanche » $1946, n^{\circ} 1,2,3$ et 6 . « Revae Générale de l'Electricité 》, Janvier 1947.

G. Evangelist : « La Regolazione delle turbine idraliche 》 (Nicola Zanichelli, Bologna 1947). de lancer ou inertie spécifique) avait plutòt tendance à diminuer, parce qu'on ne voyait pas de raison majeure de ne pas admettre des survitesses de plus en plus élevées et parce que des questions économiques (prix de l'alternateur) le voulaient aussi.

Dans ces conditions, la stabilité de marche des groupes hydrólectriques, ainsi doués d'inertic hydraulique de plus en plus grande et d'inertie mécanique de plus en plus faible, ne pouvail plus ètre assurée qu'en augmentant la lenteur de réglage (1) tachymétrique des régulateurs de quelque type qu'ils soient.

Devant le nombre de plus en plus grand des centrales ainsi donces de grandes lenteurs do réglage, on pouvait craindre que la fréquence des réseaux alimentés par elles ne se trouve mal réglée. Aussi, les turbiniers ont mis en garde les exploitants contre le danger qu'il y avait d'adopter, pour des raisons économiques ou autres, des faibles $\mathrm{P} d^{2}$ pour les alternateurs, de grandes vitesses d'écoulement dans les conduites forcées et de remplacer les chambres d'équilibre par des déchargeurs ne participant pas aux réglages de faible amplitude.

Fort heureusement, en réalité, des influences stabilisantes dues à l'inertie des moteurs utilisateurs (2) et aux caractéristiques couple fréquence des réseaux électriques (3) viennent i l'encontre des influences ci-dessus.

(1) Lors de precedents articles, nous arons appele "rapidite de reponse lio » linverse de ce temps, que W. Gaber appelle, par ailleurs, « promptitude de reglage ». En l'appelant « lenteur de réglage », nous tenons compte des réflexions échangées lors d'une recente seance de la Sté Francaise des Electriciens, a cours de laquelle, d'alleurs, M. STEN, d'Escher Wyss, a employé cette denomination.

(2) Voir notamment : Gabrex : ourage et articles cites. Alukinas : articles cités.

(3) Voil notamment : STrem : anteles cités. 
Par ailleurs, l'interconnexion, en diminuanl les variations relatives de la puissance demandée par la clientèle, ce qui permet un bon réglage de la fréquence malgré de grandes lenteurs de réglage des groupes, supprime, dans bien des cas, le danger ci-dessus mentionné, lequel n'apparầt que lorsque accidentellement un réseau so trouve séparé de l'ensemble interconnecté. (+)

\section{Lenteur de réglage}

Une caractéristique commune à tous les régulateurs de turbines à quelque type qu'ils appartiennent est done la "lenteur de réglage " (i) homogène à un temps. C'est le temps que mettrait le groupe à faire varier sa puissance do $1 \%$ sous un écart de fréquence de $1 \%$. Nous l'appellerons $\tau^{\prime}$.

Elle est obtenue dans les régulateurs accélérotachymétriques en diminuant le coefficient d'action du tachymètre el selui du tiroir de dislribution; dans les régulateurs à dash-pot, elle est obtenue par freinage de la rapidité d'action du tachymètre et du tiroir de distribution au moyen d'un asservissement à dash-pot.

Done, suivant le type de régulateur, accélérotachymétrique ou tachymétrique à dash-pot, la lenteur de réglage dépend du tachymètre et d: tiroir de distribution (dans le premier cas) et du legré d'asservissement et de la rigidité du dashpot (dans le deuxieme cas).

Cependant, mème dans ce dernier cas, le tiroir de distribution doit avoir certaines qualités et c'est pourquoi nous allons étudier plus en détail l'ensemble constitué par un tiroir de distribution et le servo-moteur quil entraine.

Nous nous bornerons à l'étude du servo-moleur a double effet.

\section{Courbe de réponse d'un tiroir de distribution :}

Nolations :

D) = diamètre du tiroir de distribution.

$\mathrm{P}_{1}=$ pression de marche de la pompe a huile $\left(H_{1}\right.$ en mètre d'huile $\left.H_{1}=P_{1} /()\right)$.

(a) $=$ poids spécifique de l'huile.

$\Delta z$ = écart du tiroir par rapport a sa position d'équilibre.

$q \quad=$ débit d'huile dans le tiroir correspondant à l'écart.

$y \quad=$ ouverture de Ia turbine.

$x=$ course du servo-moteur (entre 0 et 1 ) rapportée à la course totale $\mathrm{L}$.

$\$=$ section du servo-moteur.

(d) Voip notamment : Alubas : articles cites.
$Y=$ cylindrée du servo-moteur $Y=$ S. I.

$s=$ section efficace du diaphragme situé sur la tuyauterie d'évacuation.

$\mathrm{F}^{*}=$ effort de vannage pour l'ouverture $y$ avec les indices oet $f$ pour désigner les efforts à l'ouverture el à la fermeture.

$\mathrm{Fm}=$ effort maximum de vannage.

$W=$ travail fictif de vamnage $W=F \times L$,

$W_{m}=$ traval fictif maximum de raunage

$W_{m}=F m \times L$.

Lorsque le piston du tiroil (fig. 1) s'abaisse de $\Delta z$, l'huile passe de l'enceinte I a l'enceinte II du servo-moteur à travers l'orifice $O_{1}$ entre le piston inférieur et la lumière du tiroir ; le piston du servo-moteur se déplace vers la droite, lhuile de l'enceinte III passe dans l'enceinte IV à travers l'orifice $\mathrm{O}_{2}$ entre le piston supérieur et la lumière du tiroir. L'huile passe enfin a la pression atmosphérique à travers le diaphragme $O$ : de section efficace s.
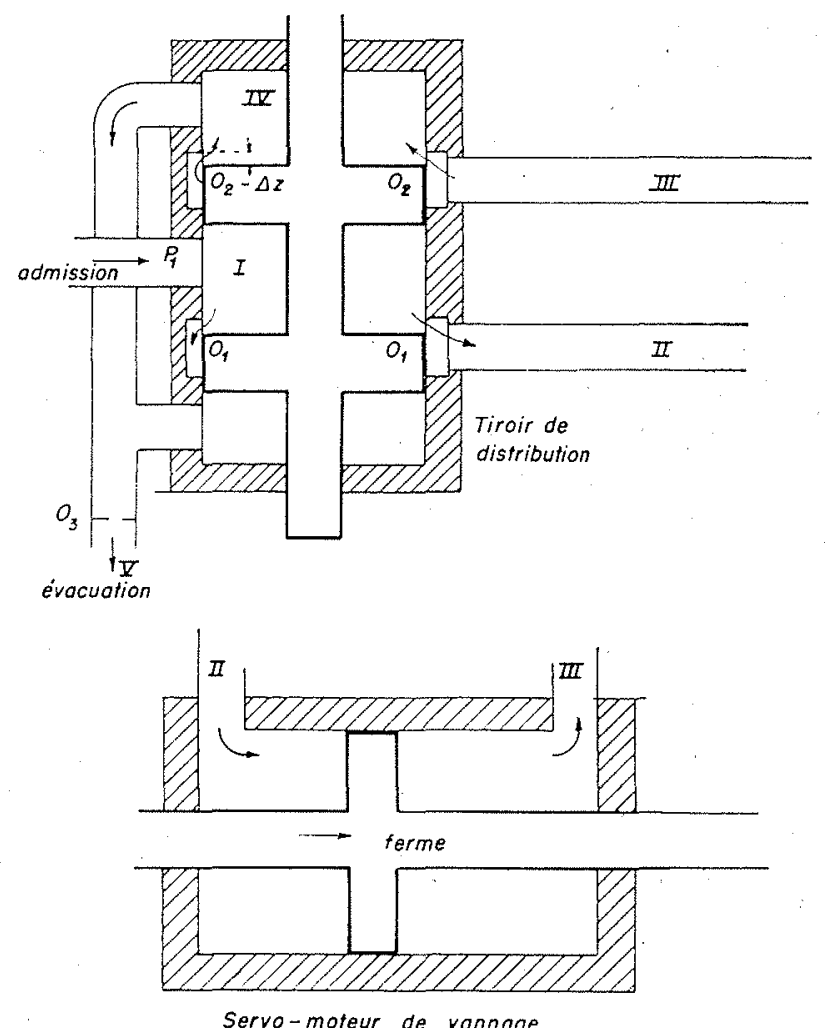

Fig. 1

Nous supposerons toutes les pertes de charge concentrées aux orifices $\mathrm{O}_{1}, \mathrm{O}_{2}, \mathrm{O}_{3}$. Nous supposerons aussi qu'à la traversée de ces orifices, l'huile perd tout son $V^{2} / 2 g$, aucune récupération n'ayant lieu à l'aval. Ceci est justifié par les dimensions donnćes habituellement aux tuyateries et au tiroir. 
Pour l'écart $\Delta z$, les orifices $O$, et $O$, ont une section efficace $\alpha \pi$ I) $\Delta z$, $\alpha$ étant un coeflicient de contraction sensiblement indépendant de $\Delta z$ el voisin de 0,6 .

On écrit que la pression de marche $P_{1}$ est égalo à la somme des pertes de charge dans les orifices $\mathrm{O}_{1}, \mathrm{O}_{2}, \mathrm{O}_{3}$, augmentée de la différence de pression entre l'une et l'autre face du piston du servo-moteur, difference de pression :

$$
\mathrm{P}=\frac{\mathrm{F}}{\mathrm{S}} \text { on } \mathrm{H}=\frac{\mathrm{F}}{\mathrm{a}} \mathrm{S}
$$

On aboutil, sans difficultés, à l'expression du débil $\%$

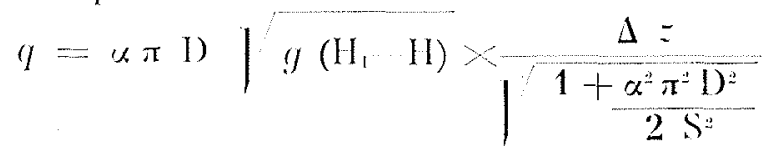

(q est consideré comme positil s'il manouve le vannage dans le sens auverture, $\Delta z$ est posilif si le piston est soulevé de $\Delta z$, une baisse (le tiroir entraînant une fermeture de vamnage).

Soit $x$ la course du servo-moteur, on a :

$$
q=\mathrm{SL} \frac{d x}{d l}=\mathrm{Y} \frac{d x}{d l}
$$

Soit y l'ouverture da vannage, comptée de 0 a 1 et définie comme proportionnelle at débit sous chute constante. La courbe $y \quad(\mathrm{x})$ dépend des liaisons mécaniques entre servo-moteur ef cercle de vamnage et de la forme de la colline de la turbine. Elle affecte la forme indiquée par la figure ci-dessous, (fig. 2).

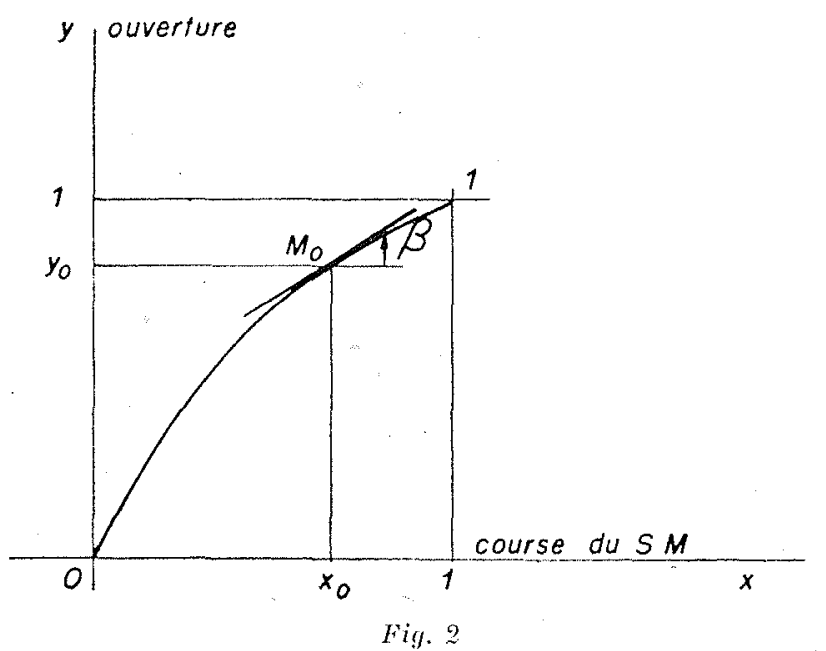

$\mathrm{Si} \beta$ est le coefficient angulaire de la tangente (qui dépend de la position du vannage) on a :

$$
\frac{d y}{d l}=\beta \frac{d x}{d l}
$$

$\beta$ est en général > 1 aux faibles ouvertures, $<1$ aux grandes et voisin de 1 à demi-charge.
Doil : $\quad q=\frac{\mathrm{V}}{\beta} \frac{\mathrm{l} l l}{\mathrm{\beta}}$

et par suite :

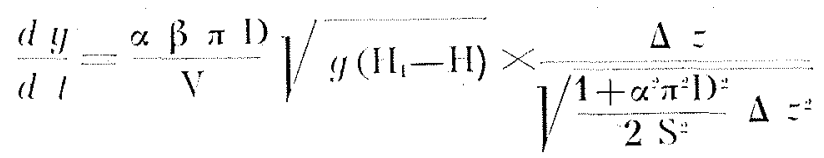

Enfin, $\Delta=$ est proportionnel à l'écart de vitesse du tachymètre, le coefficient de proportionnalité dépend de liaisons mécaniques entre lachymetre et tiroir, et de la caractéristique du tachymitre.

$$
\Delta=\ldots \mathrm{K} \Delta \omega
$$

-- otant la vitesse rapportée à la vilesse de régime.

- $\Delta$ (o) est l'ecart relatif de vitesse.

Donc: :

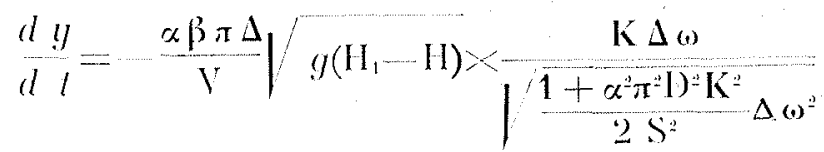
représente la loi de réponse du régulateur. C'est sa véritable loi de réponse tachymétrique dans le cas d'un régulateur accéléro-tachymétrique, mais ce n'est pas sa véritable s'il s'agit d'un régulateur à dash-pot.

La loi de réponse (1) est représentée par la courbe suivante (figure 3 ).

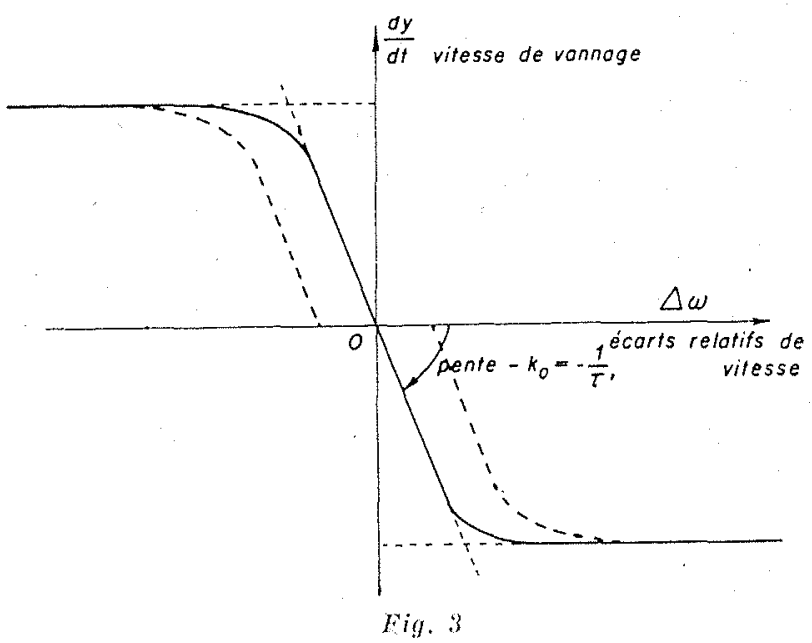

Si, clans la realite, il y avait un recouvrement du tiroir, la loi de réponse serait celle représentée en pointillé.

Cette courbe de réponse est essentiellement caractérisée par sa tangente à l'origine el par la vitesse maximum $\frac{d y}{d l}$ d'ouverture et de fermeture. La première est intéressante du point 
de vue de l'étude de la stabilité (petits mouvements de réglage, petits écarts de vitesse $\Delta \omega$ ), la deuxième du point de vue des grands mouvements de vannage (grande valeur de $\Delta(1)$ ).

a) Petits mouvements de réglage : on a au voisinage de l'origine :

$$
\frac{d y}{d l}=\mathrm{K} \frac{0,6 \beta \pi \mathrm{l})}{\mathrm{V}} / g\left(\mathrm{H}_{1}-\mathrm{H}\right) \Delta \omega
$$

Donc la lenteur de réglage, qui est par dófinilion le rapport :

$$
\mathrm{T}^{\prime}=\frac{\Delta(1)}{\frac{d !}{d l}}
$$

s'exprime par :

$$
\tau^{\prime}=\frac{V}{0,6 \mathrm{~K} \beta \pi \mathrm{l}) \sqrt{g\left(\mathrm{H}_{1}-\mathrm{H}\right)}}
$$

b) Grands mouvements sitesse maximum de vannage :

$$
\left(\frac{d \underline{l}}{d l}\right)_{m}=\frac{\beta_{s}}{\mathrm{~V}} \sqrt{2 g\left(\mathrm{H}_{1}-\mathrm{H}\right)}
$$

Ces formules ont été vérifiées expérimentalement en 1943, en collaboration avee M. CHevars. L'appareillage était très simple. Les essais avaient

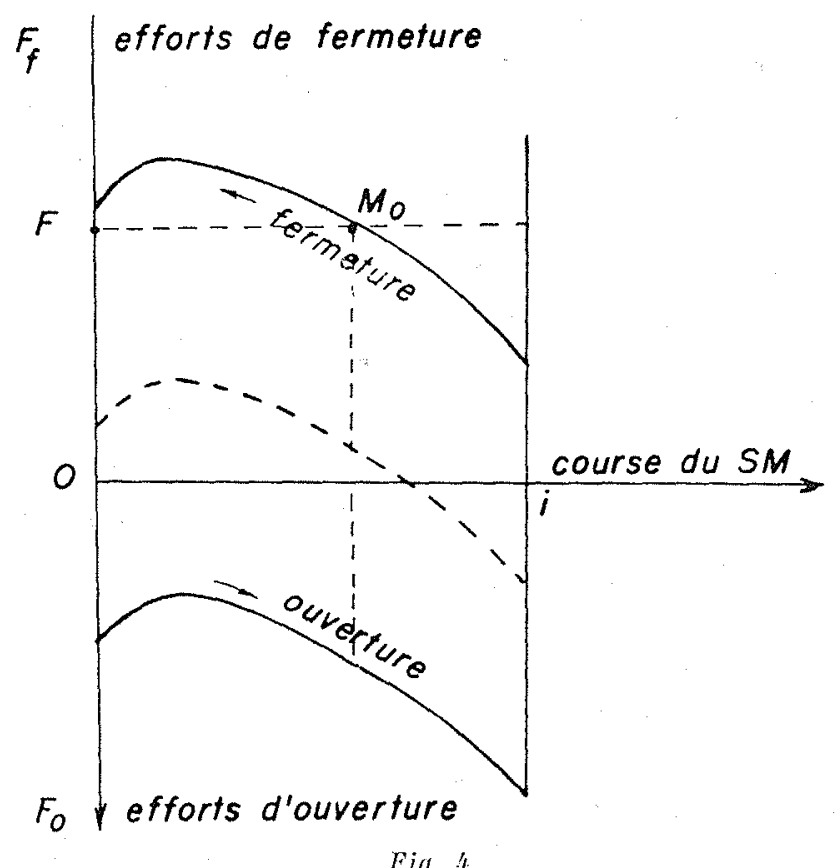

lieu en atelier. Un comparateur mesurait la position du tiroir tandis que pour un déplacement domé du tiroir, on enregistrait la loi de manieuve du servo-moteur. L'effort de vannage était matérialisé par un deuxième servo-moteur accouplé au précédent et dont on fermait l'évacuation par un orifice réglable.
On constatait notamment que lorsqu'on augmentait l'effort de vannage, la courbe de réponse «s'aplatissait par une affinité des ordonnćes (fig. 5). L'écart, à partir duquel la vitesse

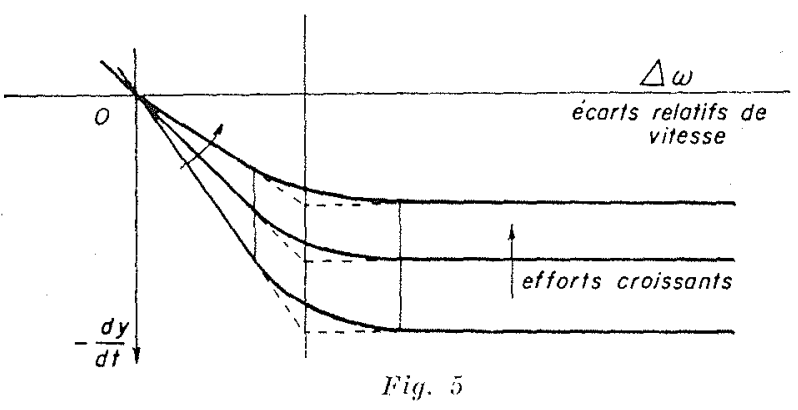

maximum de vannage ćtait alteinte, ne dépend pas de l'effort de vannage. Par contre, pour un effort de vannage donne, la diminution du diaphragme d'évacuation modifie la vitesse maximum de vannage, sans modifier la pente a l'origine, de sorte que l'ćcart au bout duquel la vitesse maximum de vannage est atteinte augmente. (Fig. (6).

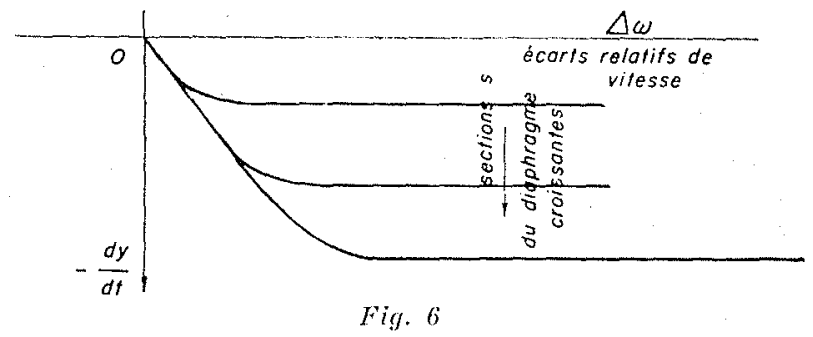

Pour une installation donnée (voir fig. 4), on peut porter en fonction de la course du servomoteur (de 0 à L) l'effort de vannage à l'ouverture et à la fermeture. On peut définir le " travail de vannage à la fermeture » et le « travil de vannage à l'ouverture $»$.

A une ouverture yo correspond un travail fictif à l'ouverture égal au produit de la course du servo-moteur par l'effort à fournir au voisinage de l'ouverture $y_{i}$ pour ouvrir le vannage. A celte mème ouverture yo correspond de même un travail fictif à la fermeture, produit de la course par l'effort de fermeture au voisinage de louverture yo.

Nous indiquerons ces travax par $W, y_{y}$ et $W_{0 y_{0}}$. Le symbole $W_{y_{0}}$ représentera le travail fictif de vannage au voisinage de l'ouverture yo, sans préciser qu'il s'agit d'ouverture ou de fermeture.

II existe une ouverture pour laquelle l'effoit est maximum. On définira donc le travail fictif maximum à la fermeture el de même à l'ouver.ture. 
Les expressions (2) el (3) deviennent, en introduisant la notion de travail de vannage:

$$
\begin{aligned}
& \mathrm{T}^{\prime}= \\
& \text { Wy" }
\end{aligned}
$$

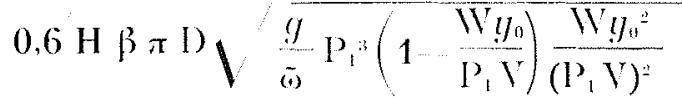

el :

$\left(3^{\prime}\right)\left(\frac{d y}{d l}\right)_{m ! n}=\frac{\beta s}{W y_{0}} \sqrt{\frac{2 g}{\sigma} \mathrm{P}_{1}:\left(1-\frac{\mathrm{W} y_{0}}{\mathrm{P}_{1} \mathrm{~V}}\right) \frac{\mathrm{W} y_{0}{ }^{2}}{\left(\mathrm{P}_{1} \mathrm{~V}\right)^{2}}}$

Pour que le servo-moteur puisse vaincre l'effort de ramnage, il faut que le produit $P_{1} V$ soit au moins égal a $W_{y}$, ceci quelle que soit l'ouverture $y ;$ il faludra donc que le produil de la pression par la cylindrée soit au moins égal au travail maximum de vannage $W^{\prime \prime}$ produit de la course par l'effort maximum. Mais cela est-il sulfisant? Evidemment non, car, pour l'ouverture $y_{m}$ correspondant a l'efrort de manouve maximum, la vitesse de vannage serait nulle et la lenteur de réglage infinie.

Ii faut donc prendre une marge. La difficulle est qu'on conmaît mal à priori les efforts de vannage. On est donc oblige de se Iaisser une cerlaine latitude pour atgmenter la pression s'il y at lieu.

Ici, le problème se pose d'une manière différente pour un accéléromètre ou pour un régulateur à clash-pot.

\section{Cas d'un régulateur accélérométrique}

Dans ce cas, les lois de stabilité obligent le constructeur à choisir une lenteur $\tau^{\prime}$ assez grande pour les petits écarts de vitesse. Par ailleurs, la vilesse maximum de vannage doil etre suffisamment grande (par la considération de la survitesse).

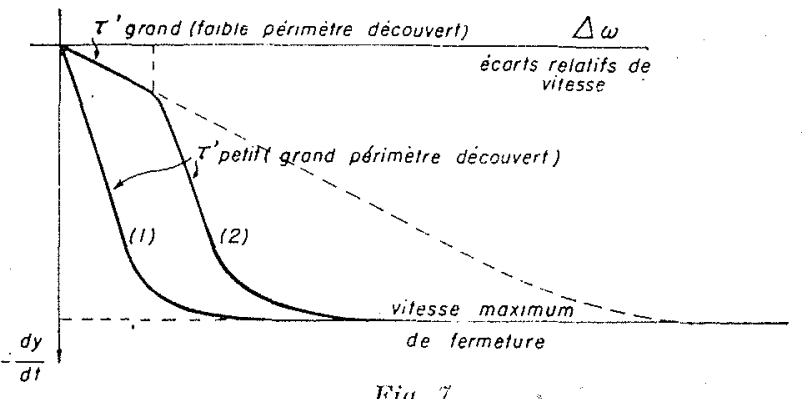

Le constructeur, pour pouvoir augmenter $\tau^{\prime}$ sans diminuer $\left(\frac{d y}{d l}\right)_{m}$ doil diminuer le périmètre al) du tiroir de distribution, mais ceci a comme conséquence d'augmenter la course du tiroir nécessaire à l'obtention de la vitesse de vannage maximum, ce qui est un inconvénient. Il s'en tirs par l'emploi du tiroir à double recouvement qui permet de ratraper, pour un écart relativement faible, la vitesse maximum de vannage (figure 7 ).
Finalement, la lenteur $\tau^{\prime}$ de réglage d'un régulateur accélérotachymétrique dépend d'abord de Ia caractéristique du tachymètre et du diamètre du tiroir, ensuite de la position du vannage (parce que le coefficient $\beta$ en dépend et parce que l'effort de vannage en dépend).

En particulier, la lenteur $\tau^{\prime}$ sera différente dans le sens ouverture et dans le sens fermeture. Pour la modifier, une fois l'installation en place, on pourra modificr la pression de marche de la pompe, on modifier la caractéristique du tachymètre et les lois de levier intermédiaires entre le tachymètre et le tiroir s'il y en a. On n'y parviendra pas en modifiant la section da diaphragme d'évacuation $s$, ce qui ne modifie que la vilesse maximum de vannage mais pas la pente de la courbe de réponse an voisinage de l'origine.

\section{Cas du régulateur à Dash-Pot: conception moderne d'un régulateur à Dash-Pot.}

Partons d'un régulateur tachymétrique direct.

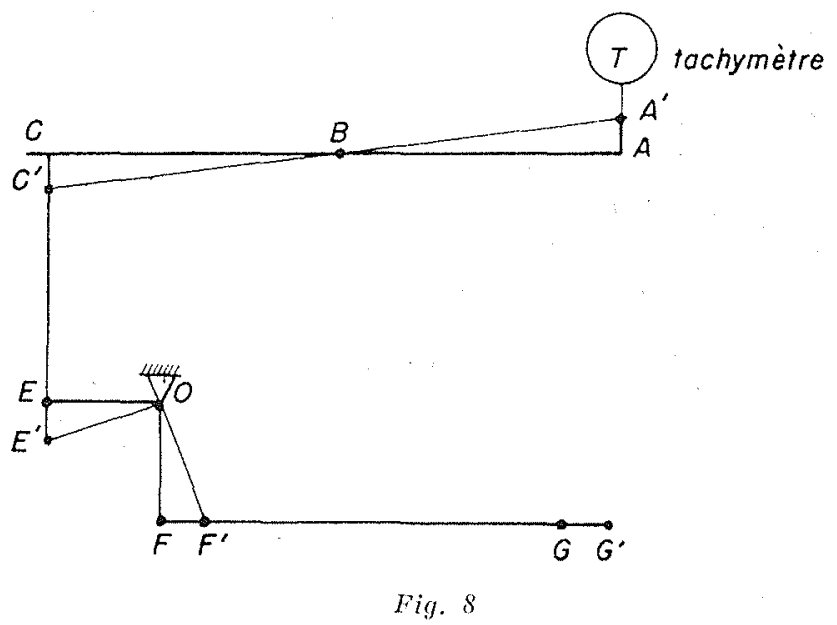

Dans le schéma de la figure 8 , supposons, c'est une vue de l'esprit, que le tachymetre soit it ce point puissant, que les déplacements de son manchon ne soient pratiquement pas affectés par les eflorts qu'il a à fournir, en sorte que la position de $\mathrm{A}$ est fonction de la vitesse seulement.

$$
\text { D) } h_{h}=K \Delta(1)
$$

Le point A entraine par le levier ABC le point Cet on a :

$$
\text { I) } \left.h_{0}=1\right) h_{\mathrm{s}}
$$

Le point C entraine le vannage au moyen d'un systeme de leviers, bielles, etc. ( $\mathrm{C}$ E F G).

On a, en fait, affaire à un régulateur « direct» puisque le vamngge est entraîné directement par le tachymetre. 
Si y est l'ourerture du vamage, on a à chaque instant :

$$
\Delta y=\frac{1}{\sigma} \Delta \omega
$$

La quantité $\sigma=-\frac{\Delta(+)}{\Delta y}$ est précisément le statisme du régulateur : écarl relatif de vitesse cutre la pleine charge et la marche a vide.

Remarquons que l'on a $\frac{d}{d l} \Delta_{y}=-\frac{1}{\sigma} \frac{d}{d l} \Delta(\omega)$, c'est-à-dire que la vitesse de vannage est proporlionnelle à l'accelération, le coefficient $\frac{1}{\sigma}$ n'étant autre que la rapidité de réponse accélérométrique $\mathrm{K}_{1}$. On voit donc que le régulateur a action directe n'est autre qu'un régulateur à loi de réponse purement accélérométrique.

Comme, pratiquement, l'efforl de vammage est trop grand pour qu'un simple tachymetre puisse le vaincre, on est amené à suspendre en $B$ un tiroir de distribution entrainant un servo-moteur, placé entre $F$ et $G$, le tout dimensionné de maniere à avoir une lenteur de réglage très laihle $\left(\tau^{\prime}=0\right)$ (fig. 9). De la sorte, pour le moindre déplacement de $B$, le vamage suit à sa vitesse maximum. Pratiquement, lors des petits mouvements de réglage, le point $B$ reste fixe, le point $C$ el par suite de rannage suit sans retard les déplacements du point A c'est-à-dire les variations de vitesse. Le régulalenr indirect asservi ainsi constitue (avec un tiroir de distribution et un servo-moteur anous d'une lenteur de reglage pratiquement nulle) se conduit exactement comme un régulateur direct lors des petils mouvements de vannage : te point $B$ reste fixe (1).

(1) On a coulume de dire qu'un régulateur direct est par essence même stable. Ce n'est vrai que lorsque le thuide motenl est sans inertic. Supposons, en effet, l'iner.. tie du fuide caractérisce par le temps $\Theta=I X / \mathrm{gH}$, cell: des masses tounantes par le temps de laneor $T$.

La condition de stabilité du régulateur a asservissemenl

$$
\frac{\tau \sigma}{\Theta}>\frac{3+\theta K_{0} \sigma}{2+\theta K_{0} \sigma}
$$

te La page 89 de la « Houlle Blanche $\gg(n " 1-19) 46)$, devient lorscue ko devient infini :

$$
\sigma>\frac{\Theta}{\tau}
$$

Or, lorsque li $o=\frac{1}{T}$, est infini, tout se passe, nous renons de le vojr, comme dans un régulateur direct.

On voit done que mème dans le réglage direct, le stittisme doit etre supericul a une valeur non nulle pour que le regiage soit stable.

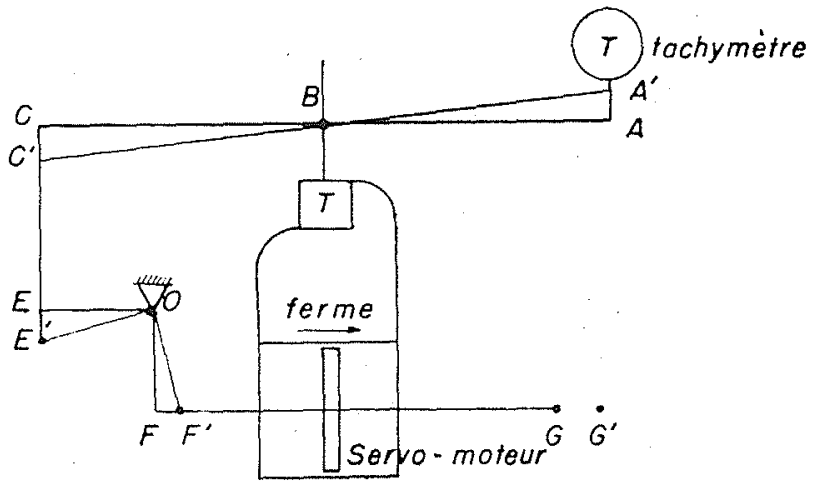

Fiq. 9

L’inconvénient du régulateur précédent étant qu'il ne règle pas à vitesse constante, l'idće vient d'interposer un dash-pot entre $C$ et $E$ et de rappeler par un ressort le point $C$ vers une position fixe (fig. 10). La encore, par suite de la lrès faible lenteur du système tiroir-servo-moteur, le point $B$ reste pratiquement fixe. Ecrirons que la vitesse du point $\mathrm{C}$ est la vitesse du point $\mathrm{E}$ et la vitesse du piston dans le cylindre ilu dash-pot :

$$
\frac{d}{d l} \Delta h_{i}=\frac{d}{d l} \Delta h_{1:}+\frac{d}{d l} \Delta h_{1}
$$

$\frac{d}{d l} \Delta h_{c}$ est proportionnel a $-\frac{d}{d l} \Delta \omega$ (accéléra$\frac{d}{d l} \Delta h_{\mathrm{r}}$ est proportionnel a $\frac{d}{d l} \Delta y$ (vitesse de enfin $\frac{d}{d l} \Delta h D$ est proportionnel à l'écart $\Delta h_{c}$, donc à $-\Delta_{(}$(vitesse).

$$
\text { Il vient : }-\frac{d}{d l} \Delta \omega=\sigma \frac{d}{d l} \Delta y-\frac{1}{\mathrm{~T}_{r}} \Delta \omega
$$

ou, par définition, $\sigma$ est le degré d'asservissement et ${ }^{r}$. la rigidité du dash-pot.

$$
\text { Doù : } \frac{d}{d l} \Delta y=\frac{1}{T_{r} \sigma} \Delta \omega-\frac{1}{\sigma} \frac{d}{d l} \Delta \omega
$$

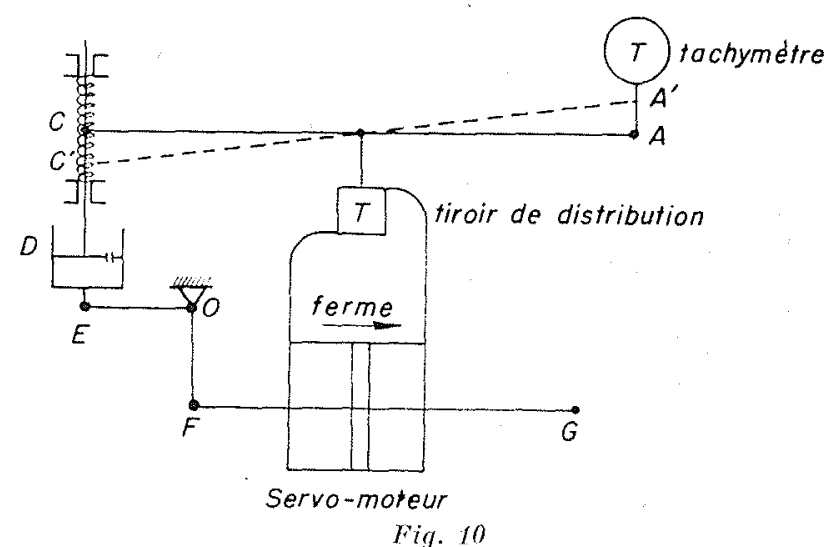


La loi de réponse du régulateur ainsi réalisé est acélérotachymétrique. La lenteur de réglago lachymétrique est $\mathrm{I} / \mathrm{Ko}=\tau^{\prime}=\mathrm{T}_{r} \sigma$.

La rapidité de réponse accélérométrique $K_{1}$ est l'inverse du degré d'asservissement

$$
K_{1}=1 / \sigma
$$

Le dosage accélérotachymétrique $m=\mathbf{K}_{1} / \mathbf{K}_{0}$ n'est autre que la rigidité ${ }^{\prime} T_{r}$. du dash-pot.

Le dash-pot et son ressort introduisent dans le régulateur direct (done purement accélérométrique) l'effet tachymétrique qui lui manquait pour légler à vitesse constante.

Nous voyons ainsi qu'il faut considérer an régulateur moderne à dash-pot non pas comme un régulatear indirect à asservissement rendu "élastique » par un dash-pot et un ressort de rappel, mais bien comme un régulateur direct : le point $B$ restant pratiquement fixe lors des petits mouvements de réglage, tout se passe. comme si c'etait le tachymètre qui entrainait le bannage.

Nous venons aussi de montrer que les réaclions d'un tel régulateur à dash-pot devant une variation de fréquence indiquée par son tachymèlre sont les mémes que celles d'un régulateur accélérotachymétrique pour lequel les variations: de fréquence se font sentir a la fois sur son lachymetre et son accéléromètre.

Dans un régulateur à dash-pot, la véritable lenteur de réglage n'est done pars celle que lui doment son tachymetre et son tiroir de distrihution; ce dernier ne sert que de relais et. comme tous les relais et pour ne pas introduire de retard, il doit ètre très rapide, c'est-á-dire qu'il faut que sa lenteur de réglage soit nulle (c'est ce qui correspond à la courbe $a_{3}=\infty$ du diagramme de la page 134 du $n^{\circ} 2$ de la "Houlle Blanche », cas exclusivement traiti par M. Gabes dans son ouvrage « Considérations... ».

Il faul done doter le régulateur d'un tiroir de distribution tel que $\tau$ ' soit le plus petit possihe ; done le diamètre du tiroir sera le plus grand possible (compatible avec les possibilités de construction). II n'y aura aucunement besoin de recourir au double recouvrement. Au contraire, le tiroir ne devra présenter aucun recouvement. Une fois le diametre du tiroir choisi, il y a lien de choisir les autres paramètres de manière quo $\tau^{\prime}$, soit le plus petit possible. L'expression (2') montre que $W_{y_{0}}$ étant une donnée de l'installation ansi que $P_{1}$, il faudra choisir la cylindree $V$ ni trop grande ni trop petite, de maniere que le produit :

$$
\left(1-\frac{\mathrm{W} y_{0}}{\mathrm{P}_{1} \mathrm{~V}}\right) \frac{\mathrm{W} y_{0}{ }^{2}}{\left(\mathrm{P}_{1} \mathrm{Y}\right)^{2}}
$$

soil maximum, éest-í-dire que :

$$
v-\frac{3}{2} \frac{W_{y_{11}}}{P_{1}}
$$

En fail, il faudra prendre:

$$
V=\frac{3}{2} \frac{W m}{P_{1}}
$$

$\mathrm{Ir}_{m}$ étant le produit de la course par l'efort maximum de vannage.

Cette valeur de $Y$ est celle qui permellait de donner au diametre du tiroir de distribution la valeur minimum nécessaire pour obtenir une lenteur de réglage donnée. Mais si cette condilion optimum nest pas respectie, on pent toujours augmenter $P_{1}$ pour diminuer $T^{\prime}$.

Il y a lien de faire le bilan entre la difficulte d'augimenter le diamètre du tiroir el celle d'ausmenter la cylindrée du servo-moteur.

En fait, on remarque que si on augmente $V$ de la valeur:

$$
\mathrm{V}=1,25 \quad \mathrm{~W}_{m} / \mathrm{P}_{1}
$$

à la valeur :

$V=1,50 \quad \mathrm{~W}_{m} / \mathrm{P}_{1}$ (augmentation de $20 \%$ de la cylindrée), on ne diminue que de $5 \%$ la lenteur de réglage, ce qui est peu payant.

Une circonstance qui fait que l'on a toujours une bonne marge entre la pression juste néces. saire pour manceuver le vanmage et celle nécessaire pour avoir une lenteur de reglage tris petite est la suivante : la pression de marche $\mathrm{P}_{1}$ est réglée par une soupape de décharge qui possède un décrément. Lorsqu'elle est onverte. débitant la totalité du débit de la pompe volumćIrique, la pression est plus forte que lorsque son débit est nul. Or, lorsque le débit de la soupape est nul, c'est que la totalite du débit de la pompe passe par le tiroir, ce qui a lieu au cours d'une manouvre à la vitesse maximum de vamnage. (Il est en effet logique de régler la tension du ressort de la soupape de manière que pendant la mancuvre à vitesse maximum, il n'y ait pas de fuite par la soupape).

Par contre, lorsque la soupape débite lout le débit de la pompe, le tiroir de distribution est au voisinage de sa position d'équilibre. De la sorte, on dispose lors des mancuvres rapides d'une pression plus faible que lors des petites mancuvres de réglage.

Si donc on a réglé la pression de manière à pouboir entrainer le vannage a la vitesse voulue, on dispose, pour les petites oscillations de rí glage, d'une marge telle que la lenteur de réglage se trouve automatiquement tres petite.

Quoi qu'il en soit, les « lenteurs de réglage» des tiroirs de distribution des régulateurs moder- 
nes à dash-pot sont comprises entre $1 / 50$ et $1 / 100$ de seconde (ce qui correspond à $K_{i}=50$ et $K_{0}=100$, dans les notations de l'article page 134 de la «Houille Blanche»).

Dans ces conditions, tout se passe comme si le relais constitué par le tiroir de distribution était sans retard, le piston du dash-pot suivant, sans retard, les indications du tachymetre (Voir Sch. Bau. M. STEn - Septembre 1947).

Ceci dit, la véritable lenteur de réglage d'ut régulateur à dash-pot n'est pas nulle.

Nous venons de voir qu'elle est égale au produit du degré d'asservissement provisoire o par la rigidite du dash-pot $\mathrm{T}$.

$$
\tau^{\prime}=\sigma^{\prime} \mathrm{T}^{\prime}
$$

On peut faire immédiatement une remarque: contrairement à ce qui se passe dans un régulateur accélérotachymétrique, la lenteur de réglag $\mathrm{T}_{r} \boldsymbol{\sigma}$ ne dépend pas de l'effort de vannage, si on a donné au tiroir de distribution des caractéristiques telles que sa lenteur soit très petite, c'esta-dire si l'on dispose d'une marge suffisante do pression au delà de celle nécessaire pour manowurer.

Pour les grands écarts de fréquence, correspondant au cas de variations importantes de charge, le diaphragme placé sur l'évacuateur. limite la vitesse de vannage à la valeur permiso par le coup de bélier.

Cependant, par suite de la petitesse nécessairo de $\tau^{\prime}=T_{r}$ o l'écart de fréquence nécessaire pour réaliser la vitesse maximum de manouve serait exagéré si l'on n'employait pas l'artifice dit « de la compensation accélérée " dont l'utilisation correspond à la même préoccupation que celui du «double recouvrement " employé dans les accélérotachymètres. Il consiste à découvrir complètement l'orifice du dash-pot lorsque l'écart de fréquence est supérieur à une certaine valeur.

Pour ces grands écarts, la valeur de $T^{\prime}$ n'est plus contrôlée par le dash-pot, c'est-à-dire assez grande, mais par le tiroir de distribution. Elle est alors très petite et dépend de l'effort de vannage.

\section{Caractéristiques optima du régulateur}

La stabilité réclame, de la part du régulateur, une lenteur de réglage tachymétrique suffisamment faible.

$$
\tau^{\prime}=\mathrm{T}_{r} \sigma<K \frac{\theta^{\prime}}{\mathrm{T}}
$$

Le coefficient $K$ dépend du paramètre d'Arlmevr, et d'une foule d'autres caractéristiques telles que la caractéristique couple fréquence du réseau. Mais il dépend aussi de caractiristiques internes du régulateur, notamment du degré d'asservissement dans les systëmes à dash-pot et de la rapidité de réponse accélérométrique (dans les accélérotachymètres).

II existe pour une installation donnce, une valeur optima du degré d'asservissement - ou de la rapidité de réponse automatique -, pour laquelle la stabilité est réalisce au prix de la lenteur de réglage minimum.

Posons (figure 11), en fonction du rapport $\frac{\theta}{\tau \sigma}\left|=\frac{\theta K_{1}}{\tau}\right|$ d'une part le rapport $\frac{\tau \tau^{\prime}}{\theta 2}$

d'autre part $\frac{\theta}{\mathrm{T}_{r}}\left|=\frac{\theta}{m}\right|$

$\frac{\theta}{\tau \sigma}$ caractérise done le degré d'asservissement rapidité de réponse accélérométrique].

$$
\begin{aligned}
& \quad \frac{\theta}{T_{r}}\left|=\frac{\theta}{m}\right| \begin{array}{l}
\text { caractérise la rigidité du dash- } \\
\text { pot (ou le dosage accélérota- } \\
\text { chymótrique) }
\end{array} \\
& \frac{\tau \tau^{\prime}}{\theta^{2}}\left|=\frac{\tau \sigma \mathrm{T}_{r}}{\theta^{2}}\right| \begin{array}{l}
\text { caractérise la lenteur de ré- } \\
\text { glage tachymétrique. }
\end{array}
\end{aligned}
$$

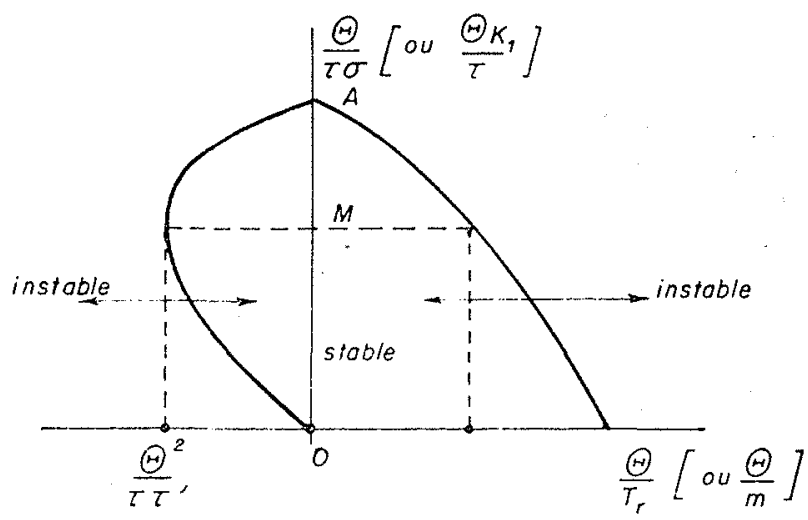

Fig. 11

Le point A caractérise un régulateur pour lequel la rigidité du dash-pot est infinie ou le dosage accélérotachymétrique est infini. Il s'agil done d'un régulateur à asservissement rigide ou accélérométrique pur.

Soit $M$ le point correspondant at maximum du rapporl $\frac{\theta^{*}}{\tau \tau^{\prime}}$ done au réglage optimum.

Si l'on a :

$$
\frac{\theta}{\mathrm{T \sigma}}\left\lceil=\frac{\theta K_{1}}{\tau} \mid \equiv \overline{\mathrm{OA}}\right.
$$

il y aura instabilité quel que soit le réglage choisi.

Si on augmente $\sigma$ (ou si on diminue $K_{1}$ ), on 
voit que la lenteur de réglage nécessaire à la stabilité décroît d'abord jusqu'à :

$$
\frac{\theta}{\tau \sigma} \Gamma=\frac{\theta \mathbf{K}_{i}}{\tau} \Gamma=\overline{\mathrm{OM}}
$$

puis augmente ensuite, si on continue à augmenter le degré d'asservissement (ou à diminuer la rapidité accélérométrique).

Le problème se pose donc de la manière suivante :

a) pour un constructéur de régulateurs à dashpot, choisir un degré d'asservissement temporaire voisin de la valeur optimum, done suffisamment supérieur à celui correspondant au point A (asservissement rigide), mais pas trop grand ; ceci se fait par le choix de la pente de la came d'asservissement par exemple. Ensuite donner au dash-pot la rigidité minimum nécessaire à la stabilité. Le choix d'un degré d'asservissement trop faible obligerait à augmenter la rigidité du dash-pot; et par suite la lenteur du réglage $T_{,} \sigma$, au delà de ce qui serait nécessaire si le choix avait été mieux fait.

b) pour un constructeur de régulateurs accélérotachymétriques, choisir une rapidité accélérométrique $K$, correspondant à l'optimum OM, et, ensuite, régler la lenteur de réglage à une valeur assez faible pour atteindre la stabilité. Le choix d'une rapidilé de réponse accélérométrique trop grande ou trop petite obligerait à augmenter la lenteur de réglage au delà de ce qui serail nécessaire si le choix de $K_{1}$ avait ble mieux fait.

\section{Mesure de la lenteur de réglage}

Il s'agit de tracer, pour chaque position du vannage, la courbe de réponse donnant, pour chaque ecart de fréquence, au voisinage de zéro, la valeur de la vitesse de variation de puissance.

La méthode préconisée et utilisée par M. GADEN sur des régulateurs accélérotachymétriques est la suivante :

- Le groupe est couplé sur le réseau à fréquence constante.

Son ouverture est : $y_{\%}$. Le réglage chargevitesse a été préalablement étalonné et gradué en écarts de fréquence. Le point de fonctionnement est le point $\mathbf{M}_{0}$, le la figure 11 .

Donnons au réglage charge-vitesse un déplacement correspondant à un écart de fréquence $\Delta \omega$.

L'accéléromètre n'agit pas, la fréquence du réseau étant constante.

La caractéristique de statisme passe de $A_{11} A_{1}$ à $A^{\prime}{ }_{1} A_{1}^{\prime}$ (fig. 11). Le régulateur est sollicité au début par l'écart de fréquence.

$$
\Delta \omega=M_{0} \mathbf{K}
$$

Son vannage va commencer par fermer à la vitesse $\frac{d y}{d t}=-\frac{\Delta \omega}{\tau^{\prime}}$ correspondant à l'écart $\Delta \omega$. Au fur et à mesure de la fermeture, l'écart qui le sollicite diminue jusqu'à ce qu'il ait atteint l'ouverture $y^{\prime}$ où l'écart est nul (r). La vitesse de fermeture diminue donc jusquà s'annuler en $\mathrm{M}^{\prime}$.. En enregistrant la mancuvre, en ne retenant de l'enregistrement que le début et en le répétant pour diverses valeurs de $\Delta \omega$, on tracera la loi de réponse autour de l'ouverture $y$ et on en tirera la lenteur de réglage par la mesure de la pente de la tangente à l'origine.

On devra répéter l'opération pour chaque valeur initiale de l'ouverture, pour avoir la valeur de la lenteur ${ }^{\prime}$ correspondant à chaque portion initiale du vannage.

Que mesurerait-on en opérant de même dans le cas d'un régulateur à dash-pot?

En fait, que se passe-t-il lorsque sur un tel régulateur, on manouvre brutalement le réglage charge-vitesse, le faisant passer d'une position à une autre correspondant à un écart de fréquence négatif par exemple? Si le statisme est nul, on constate que le vannage ferme d'abord pendant un court instant, à la vitesse maximum, celte vitesse ralentit ensuite jusqu'à une valeui praliquement constante, cela jusqu'à la pleine fermeture. Si le statisme n'est pas nul, le début du mouvement est le mème, d'abord vitesse maximum de vannage, très vite freinée jusqu'à une certaine vitesse, mais ensuite diminution très lente de cette vitesse jusqu'à l'arrêt à la nouvelle position $y{ }^{\prime \prime}$ ".

Comment peut-on expliquer ce comportement? Il faut se rappeler que la lenteur de réglage est contrôlée par le degré d'asservissement provisoire et le dash-pot. Lorsque la rigidité devient infinie, le degré d'asservissement provisoire

(1) On pent falre la remarque suivante : Sous un ecart initial du réglage de charge-vitesse, le régulateur va mancuvrer son vannage à la vitesse :

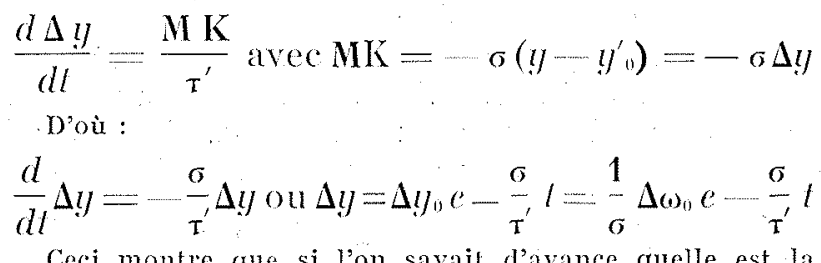

Ceci montre que si lon savait d'avance quelle est la modification du réglage charge-vitesse qu'il fant poul' faire passer le vamage de louverture yo à l'ouverture $y^{\circ}$, et qu'on la réalise dès le début, le temps que le régulateur met pratiquement à manœuvrer de yo à 'y'o ne dépend pas de la distance à parcourir le temps' au bout duquel le régulateur a parcouru une fraction donnée). 
devient un statisme permanent beaucoup plus élevé que le statisme normal. La lenteur de réglage $\tau^{\prime}=T_{r} \sigma$ devient infinie. Mais cela ne veut pas dire que le vannage ne se déplacera pas sous un écart de fréquence $\Delta \omega$.

Considérons, en effet, un régulateur de statisme nul et rendons permanent son statisme temporaire en obturant complètement l'orifice du dashpot $\left(T_{r}=\infty\right)$. Nous aurons donc une caractéristique de statisme $A_{0} A_{1}$ très inclinée (pente égale au statisme temporaire) passant par le point de fonctionnement $M_{0}$. (fig. 12). Si nous donnons au réglage charge-vitesse un écart correspondant à l'écart de fréquence $\Delta \omega$, cette caractéristique devient A' $A^{\prime}$. Ce point de fonctionnement passe de $\mathrm{M}_{\text {"à }} \mathrm{M}^{\prime}{ }_{0}$. Il est sollicité au début par l'écart de fréquence $\Delta \omega=\mathrm{M}_{0} \mathrm{~K}$ et, à la fin, par un écart nul, mais comme la lenteur de réglage du tiroir de distribution est, ici, très petite, c'est-à-dire que le moindre écart de fréquence déplace suffisâmment le tiroir pour atteindre la vitesse maximum de vannage, le vannage passera de l'ouverture $y_{0}$ à l'ouverture $y^{\prime} o$ à la vitesse maximum, le mouvement n'étant freiné que tout à fait à proximité de M'o.

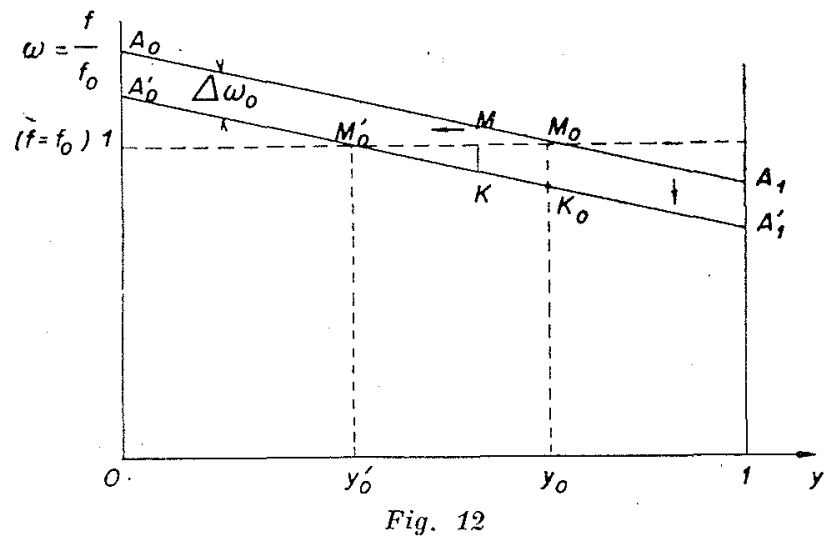

Recommençons l'expérience à partir de l'ouverture $y^{\prime}$ a avec un statisme nul, après avoir légèrement débouché l'orifice du dash-pol. de manière à rendre $\tau^{\prime}=T_{r} \sigma$ fini.

On comprend, dès lors, que le régulateur vi fermer à la vitesse maximum jusqu'à une ouverture voisine de $y^{\prime} o$, tout se passant pratiquement comme si l'asservissement temporaire était permanent durant ce mouvement. Le servomoteur fermera ensuite lentement à la vitesse $\frac{d x}{d t}=\frac{\Delta \omega}{\mathrm{T}_{r} \sigma}$ correspondant à celle d'un régulateur de lenteur $T, \sigma$ sollicité par un écart do fréquence $\Delta \omega$.

S'il y a un statisme permanent, le régulateur. au lieu de fermer complètement le vannage, s'arrêtera à l'ouverture $y_{0}^{\prime \prime}$, correspondant au point M"O intersection de l'ordonnée de fréquence fo avec la caractéristique de statisme permanent A" $A$ ".

Entre les ouvertures $y^{\prime}$ el $y^{\prime \prime}$, la vitesse du servo-moteur diminue linéairement de $\frac{d x}{d l}=\frac{\Delta \omega}{T_{r} \sigma}$ à 0 . Ainsi, se trouvent expliquées les constatations faites plus haut.

Remarquons que ce comportement du régulateur à dash-pot n'est pas sans dérouter les gens chargés de les manipuler.

Il arrive, en effet, que si un dash-pot est trop serré ( $T_{r}$ un peu grand), l'agent chargé de mancuvrer la tirette charge-vitesse, pour faire augmenter la puissance de $\Delta P$ kilowatts, envoie une première impulsion, constate sur un wattmètre que la puissance varie rapidement d'une quantité $\Delta P$, Kw inférieure à l'augmentation demandée, puis varie très lentement.

Croyant que cette lenteur est l'indice qu'il va s'arrêter à une puissance trop faible, il donne, impatient, une autre impulsion, le vannage fait un nouveau saut rapide et la puissance aboutit rapidement à la puissance demandée. Croyant avoir fait son devoir, l'agent retourne à ses occupations normales, pour s'apercevoir avec stupeur au bout d'un moment que la puissance est beaucoup trop grande. Le régulateur a continué à fermer à la vitesse correspondant à celle d'un régulateur de lenteur normale sollicité par la double impulsion donnée au réglage chargevitesse.

Placé avec la même consigne devant un régulateur accélérotachymétrique, l'agent aurait donné une première impulsion, aurait constaté que le régulateur ouvrait très lentement; il en aurait ensuite donné d'autres pour le faire aller plus vite. Mais il aurait alors constaté qu'il dépassait la puissance demandée et il aurait été condurit à donner des impulsions en sens inverse pour l'atteindre.

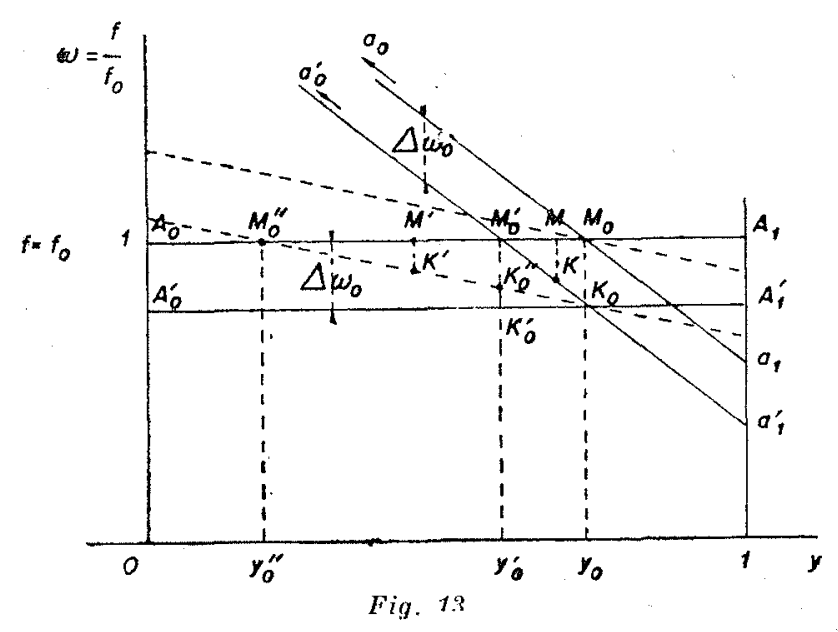


Bien entendu, dans ce cas comme dans l'autre, un agent ayant l'habitude de ce travail et connaissant bien son régulateur arrive assez facilement à la nouvelle puissance demandée par le programme.

Mais ceci est assez troublant. N'a-t-on pas vu, en effet, que les deux systèmes de régulateurs sont équivalents? Comment se fait-il alors que, soumis à l'action du réglage charge-vitesse, le régulateur accélérométrique démarre lentement dès le début avec sa lenteur normale, alors que le régulateur à dash-pot, réputé plus lent, démarre à toute allure, son ardeur étant ensuite rapidement freinée par son dash-pot jusqu'à sa lenteur normale.

La réponse est facile : si l'équation différentielle qui régit le mouvement est la même dans les deux cas, les conditions initiales ne sont pas les mêmes. Dans le régulateur accélérotachymétrique, le déplacement du réglage charge-vitesse n'introduit aucun effet accélérométrique puisque le régleur est maintenu à vitesse constante par le réseau. Dans le régulateur à dash-pot, l'effet accélérométrique initial existe d'une manière un peu cachée, il est vrai, mais réelle, se traduisant par le fait qu'au début le mouvement de vannage n'est pas freiné par le dash-pot.

Quoi qu'il en soit, si on appliquait au régulateur à dash-pot, la méthode ci-dessus pour la mesure de la lenteur de réglage, (enregistrement du début de la manœuvre après manœuvre du réglage charge-vitesse), on ne mesurerait pas la véritable lenteur de réglage, contrôlée par le dash-pot $\left(\tau^{\prime}=T_{r} \sigma\right)$, mais la lenteur de réglage beaucoup plus faible du tiroir de distribution. Pour lever cette difficulté, on pourrait, par exemple, mettre le statisme à zéro et enregistrer la loi de fermeture de la pleine charge à la marche au vide, ou d'ouverture de la marche à vide à la pleine charge, sous l'action d'écarts fictifs de fréquence $\Delta\left(\omega_{1}, \Delta \omega_{*}, \Delta \omega_{; ;}\right.$, etc. donnés par le réglage charge-vitesse.

Il faudrait laisser délibérément de côté le début de chacune de ces courbes et n'utiliser que les parties correspondant à des déplacements lents, d'où on tirerait la valeur de $\tau^{\prime}=\mathrm{T}_{r} \sigma$ pour chaque ouverture. Notons, d'ailleurs, qu'ici la valeur de $\tau^{\prime}$ ne dépend pas de l'effort de vannage, mais uniquement de $\mathrm{T}_{r}$ et de $\sigma$; comme on connaît la valeur de $\sigma$, pour chaque ouverture, notamment d'après la pente de la came et la loi ouverture-course du servo-moteur, il suffira de connaître " $T$ ' $=T_{r} \sigma$ à une ouverture quelconque pour en déduire sa valeur à n'importe quelle ouverture.

\section{CONCLUSION}

Nous pensons avoir fait cuvre utile en précisant les différents problèmes que doivent résoudre les constructeurs de régulateurs de turbines hydrauliques pour fournir à leurs clients des régulateurs remplissant leurs fonctions dans les conditions optima compatibles avec l'installation, qu'ils contrôlent.

Nous avons montré que les tiroirs de distribution de régulateurs accélérotachymétriques et iachymétriques à dash-pot ont des caractéristiques qui répondent à des préoccupations tout à fait différentes.

Dans le premier cas, le tiroir de distribution conditionne la lenteur de réglage, caractéristique essentielle du régulateur.

Dans le deuxième cas, le tiroir de distribution n'est pas une chose qui va de soi et nous avons essayé de la préciser.

Il est pottrtant utile de se rappeler que le régulateur à dash-pot est, malgré tout, un régulateur à statisme rendu temporaire pour expliquer certaines particularités de son comportement. 